\title{
Knowledge, Awareness And Practice of Sudanese Dentists Towards Oral Radiology And Protective Guidelines
}

\author{
Rouwan Elfatih Hussein $^{1}$, Nada Tawfig Hashim ${ }^{2}$, Elhadi Mohieldin Awooda ${ }^{1 *}$ \\ ${ }^{I}$ Department Of Conservative Dentistry: Faculty Of Dentistry, University Of Medical Sciences And Technology, \\ Sudan*. \\ ${ }^{2}$ Department of Periodontics: Faculty Of Dentistry, University Of Medical Sciences And Technology, Sudan \& \\ Department of Periodontology: Faculty of Dentistry, University of Khartoum, Sudan \\ *Corresponding author: Dr. Elhadi Mohieldin Awooda: sowaraldahb@gmail.com
}

\begin{abstract}
:
Background: In dentistry, radiographs are very helpful in detecting oral diseases, cavities, bone related problems, treatment planning as well as follow-up treatments. However, it should be clinically justified to obtain maximum benefit and reduce further effects. This study was conducted to assess the knowledge, awareness and practice of general dental practitioners towards oral radiology and protective guidelines.

Materials and Methods: A descriptive cross - sectional study carried out among 198 general dental practitioners working within Khartoum locality during period from January 2016 - April 2016. Data was collected via self administered questionnaires composed of demographic data and closed ended questions regarding their knowledge, awareness and practice towards the hazardous effects of dental x-ray and radiographic protection guidelines. Comparison between variables was assessed by Chi square test with the level of significance set at $P \leq 0.05$.

Results: The overall correct response among the study population was ranged $26.3 \%-74.3 \%$. more than half (55.1\%) were aware that dental $x$-rays are harmful, $40.9 \%$ know that $x$-ray beams cannot be reflected from room walls, $48.5 \%$ perceived that $x$-rays do cause ionization to matter, while $46 \%$ had the knowledge of deterministic and stochastic effects and the majority $74.3 \%$ recognized that dental radiography is not an absolute contraindication in pregnant women. Significant correlation between the number of years in practice and level of knowledge and practicing protective measure regarding radiation exposure $(P<0.05)$, but no difference were found between male and female dentists $(P<0.05)$.

Conclusion: The results indicated insufficient knowledge and awareness regarding $x$-ray exposure, and practice of protective measures. This outcome necessitates continual education by way of dental radiology courses to ensure maximum safety for both dentist and patient.
\end{abstract}

Keywords: dental radiography, hazard of oral X-ray, Sudanese dentist, $x$-ray exposure

\section{Introduction}

Radiation is the energy that transmits through space and matter. These radiations could either be from a natural source" external or internal sources" or a man-made source [1] that mostly contributes to medical and dental radiography.[2] In dentistry, radiographs are very helpful in detecting oral diseases, cavities, bone related problems, treatment planning as well as follow-up after treatments.[3] Oral and maxillofacial radiographic imaging can either be done conventionally "e.g. intraoral, extra oral and occlusal radiography" or by using specific imaging technique "e.g. tomography, CT scan and MRI.[4]

Regardless of the type of X-rays we use, problems arise due to their misuse and those may include patient related effects. These harmful biologic changes can either affect the cells by directly striking the cells and cause alteration of the structure and function of the affected molecules or indirectly by formation of free radicals which react with other free radicals resulting in formation of hydrogen peroxide which can break down large molecules such as proteins and DNA and cause cell damage. [5]

According to the probability of occurrence, biological hazards are classified into: deterministic "nonstochastic" and stochastic effects. Deterministic effects occur when there is a certain dose if exceeded then harmful insults start to appear. Stochastic effect meaning that the amount of exposure doesn't reach the determined dose so there would be no damaging insults. [6] These biological changes can lead to: skin injuries, changes in the blood forming organs and nature of the circulating blood cells, cancers, genetic effects and cataract formation.[3]

Even though dental radiation exposure is considered as minimal, the dentist should evaluate the patient's need and insure that the patient will have the maximum diagnostic benefit and strictly follow the ALARA "as low as reasonably achievable" principle as recommended by The International Commission on Radiological Protection (ICRP).[7] Regarding the risks of $\mathrm{x}$-ray exposure of the patients, staff and operator, 
proper protective measures should be taken to reduce damaging effects. Recently, newer technologies have introduced like the use of digital radiography which is rapidly increasing and is now coming to the fore. However, direct digital radiographic use is of a great potential in the dental practice and can be used for clinical and diagnostic benefits, referral purposes, distinct environmental advantages and most importantly lowered radiation dosages.[8,9] In addition, unnecessary radiation exposure should be prevented by the use of lead aprons, thyroid protectors, gonad shields, fast films, film holders and alternative methods for caries detection, which are highly recommended for children, women of childbearing age and pregnant women.[10]

Dentists as health care providers are responsible for prescribing and exposing patients to dental radiographs. Since dental radiographic examination is widely used in dentistry, public health concern should be given to its hazardous effects.[11] Therefore they should have a thorough knowledge about X-ray exposure and be strictly following the ALARA "as low as reasonably achievable" principle as to protect the patient from any unnecessary radiation exposure that may lead to irreversible damage.

The objectives of this study were to assess and compare between male and female, newly graduates and experience dentists regarding knowledge and awareness towards the risk of X-ray exposure and commonly used protective measures. Also to assess their practice of the concept of "as low as reasonably achievable" X- ray radiation exposure

\section{Materials and Methods}

A descriptive cross-sectional study conducted in Khartoum locality, one of the seven localities comprising Khartoum State, which is the capital of Sudan. The study was carried during the period from January 2016 to March 2016 among general dental practitioners working in public hospitals. The sample size was 198, obtained from the total number of 400 general dentists registered in Sudan Medical Council and practicing within the locality in four hospitals. Those who involved in administrative job, specialists or dental students were excluded. Participants were selected randomly from four public hospitals in the locality. Selection was made according to their names and registrations number in medical council by using random table.

Self-administered, close ended questionnaire was used for data collection. Questionnaire was designed according to Prabhat et al.,[6] with some modifications to suit Sudan context. The questionnaire was sectioned into three parts:

Part one: Demographic data consists of 4 items “age, gender, post-graduation experience and working area".

Part two: Assessment of knowledge, awareness and practice towards the hazardous effects of dental xray, this part consisted of 5 items with one response per each item either yes, no or I don't know.

Part three: Assessment of knowledge, awareness and practice regarding the radiographic protection guidelines, this part contained 7 questions with one response per each item either yes, no or I don't know. The questionnaire was pre tested by 20 randomly selected general dentists to ensure comprehensibility, reliability, relevant and accuracy in the Sudan context. Cronbach's alpha test showed the reliability coefficient of 0.86 and found satisfactory for conducting the study. These 20 questionnaires were not included in the final study.

Questionnaires were distributed to the selected dentists by one of the researchers and were handled early morning during the working days and handed up at the end of day. Eligible dentists were requested to participate voluntary and they signed informed written consent.

Data were entered in Microsoft Excel and transferred into SPSS (version 17; SPSS Inc., Chicago, IL). Findings were presented as group proportions, and difference in proportions for a given factor was assessed by the Chi-square test. A $P$ value cut off for statistical significance was set at 0.05 . The study was approved by the ethical research committee of University of Medical Sciences and Technology and the eligible dentists were requested to participate voluntary. An informed written consent was obtained. Names and any personal related data were kept confidential and were only used for the purposes of the study.

\section{Results}

The study participants included 198 subjects. Majority graduated less than 2 year (69.7\%), while $30.3 \%$ had graduated over 2 years.

Regarding knowledge, the overall correct response ranged from $45.5 \%$ to $74.2 \% .55 .1 \%$ were aware that dental x-rays are harmful, $40.9 \%$ know that x-ray beams cannot be reflected from room walls, $48.5 \%$ perceive that $\mathrm{x}$-rays do cause ionization to matter, while $46 \%$ had the knowledge of deterministic and stochastic effects and the majority $74.3 \%$ recognize that dental radiography is not an absolute contraindication in pregnant women (Table 1)

As far as radiographic protection guidelines are concerned, $29.8 \%$ were aware of NCRP and ICRP, while $26.3 \%, 51.5 \%$ were familiar with the ALARA principle and collimators and the uses of filters in dental radiography. Additionally $66.7 \%$ were conscious and $41.4 \%$ were knowledgeable about the fact that digital 
radiography requires less radiation exposure than conventional and that high speed films reduce exposure. Also, $76.8 \%$ prefer not to hold the films during exposure and $20.7 \%$ use lead aprons regularly.

From the results obtained there was no significant relationship between males and females regarding the knowledge about the harmful effects of dental x-rays, room walls being reflective to $\mathrm{x}$-ray beams, deterministic and stochastic effects and pregnant women $\mathrm{x}$-ray exposure? While a significant level of association between gender and knowledge about matter ionization by $\mathrm{x}$-ray was found, with males being more knowledgeable than females $(\mathrm{P}=0.01)$ as shown in Table 2 . According to the result findings, there was a significant correlation between knowledge of general dental practitioners about $\mathrm{x}$-ray exposure and years of experience (Table 3$)$.

\section{Discussion}

The present study was conducted to assess Knowledge, awareness and Practice towards Oral Radiology and protective guidelines among Sudanese males and females dentists of different years of experience with the response rate of $100 \%$ similar to others.[12,13] To our knowledge no previous studies focusing on radiation safety have been conducted in the Sudan.

Dental radiation is considered of small dose; however cumulative exposures may lead to irreversible damage. Previous studies have shown increased occurrence of thyroid cancer, salivary gland tumors and intracranial meningioma,[11] but this is not yet confirmed and not applied to the dental radiation. The risk of causing stochastic effects which can occur with minimal doses [6] should not be overlooked. Thereby; In order to prevent such effects a thorough knowledge about $\mathrm{x}$-ray exposure and protective measures is mandatory.

The results demonstrated that the majority of the studied population considered dental $\mathrm{x}$-ray to be harmful, yet a percentage of $55.1 \%$ was not promising of a safe future practice. However, this result was similar to the results obtained by Eman \&Arnout 2014.[6] The results also showed that less than 50\% of the respondents knew that $\mathrm{x}$-ray beams do reflect from room walls. This result indicates that there is a certain lack of basic $\mathrm{x}$-ray knowledge similar to others.[7,12-16]

Regarding pregnant women, a pleasing result was revealed, as most of the involved dentists recognized that dental radiography is not absolutely contraindicated in pregnant women. This result was better than those obtained by Saudi study [6] and Tabriz [17] which indicated insufficient knowledge. Despite that, dental radiographs should not be prescribed to pregnant women unless necessary.[17]

The study also conveyed that only $46 \%$ were aware of deterministic and stochastic effects of radiation while the rest were unaware of the possible biological hazards of $\mathrm{x}$-ray exposure. In addition, less than one third of respondents were aware of ALARA principle. This was a disappointing result which was most likely due to insufficient undergraduate education. Lead aprons are patient-protective equipment that reduces scattered radiations. Unfortunately, only $20.7 \%$ used lead aprons on a regular basis which was almost similar to that obtained from study in Korea with a percentage of $21.7 \%$, [11] and also agrees with a related study carried out among the Belgian dentists manifesting that $88 \%$ of dentists did not use lead aprons regularly.[18] This might be due to a lack of strict regulations regarding safe practice [19] or unavailability of lead aprons. In general, the results reflected poor awareness toward x-ray exposure and precautions, similar results revealed from others, $[1,3,10]$ but from the literature; one study concluded that radiation protection measures were more significant amongst clinical specialists.[20]

In addition, a significant correlation between gender and awareness of usefulness of collimators and filters in dental radiography was noted, with females being more aware than males. The same association was reported in the Belgian study [18] and this may explained that female dentists might have experienced the use of lead aprons during pregnancy. However, this might also be attributed to the caring nature of females.

Moreover, dissimilar to the results obtained in Tabriz, [17] our results indicated that there was a relationship between the number of years in practice and the awareness about the exposure hazards and protective measures in the majority of questions, which is similar to Prabhat et al result.[21] This means the more the dentist exposed to practice the more knowledge and protective measures acquired.

\section{Conclusion}

There was an overall lack of knowledge among Sudanese general dental practitioners working within Khartoum locality; regarding radiation exposure and protective guidelines. However, there was a highly significant correlation between number of years in practice and the level of knowledge regarding both radiation exposure and protective measures. Education regarding oral radiology biological hazards and practice safety is recommended in both undergraduate and postgraduate levels.

\section{References}

[1]. Praveen B, Shubhasini A, Bhanushree R, Sumsum P, Sushma C. Radiation in dental practice: awareness, protection and recommendations. J Contemp Dent Pract. 2013;14:143-8 
[2]. Sources and Effects of Ionizing Radiation: United Nations Scientific Committee on the Effects of Atomic Radiation. UNSCEAR 2000 Report to the General Assembly, with Scientific Annexes. volume 1: Sources. ISBN 92-1-142238-8.

[3]. Binnal A, Rajesh G, Denny C, Ahmed J, Nayak V. Insights into the state of radiation protection among a subpopulation of Indian dental practitioners. Imaging Sci Dent 2013;43:253-9.

[4]. Shah N, Bansal N, Logani A. Recent advances in imaging technologies in dentistry. World J Radiol 2014; 6: 794-807

[5]. Lobo V, Patil A, Phatak A, Chandra N. Free radicals, antioxidants and functional foods: Impact on human health. Pharmacognosy Reviews. 2010;4:118-126.

[6]. Arnout E, Jafar A. Awareness of Biological Hazards and Radiation Protection Techniques of Dental Imaging- A Questionnaire Based Cross-Sectional Study among Saudi Dental Students. J Dent Health Oral Disord Ther. 2014;1:1-8.

[7]. Arnout E. Knowledge, Attitude and Perception among Egyptian Dental Undergraduates, Interns and Postgraduate Regard Biological Hazards and Radiologic Protection Techniques: A Questionnaire Based Cross-Sectional Study. Life Sci J 2014;11: 9-16.

[8]. Wenzel A, Gröndahl H. Direct digital radiography in the dental office. Int Dent J. 1995;45:27-34.

[9]. Parks ET, Williamson GF. Digital Radiography: An Overview. J Contemp Dent Pract 2002;3:23-39

[10]. Abbott P. Are dental radiographs safe? Australian Dental Journal 2000;45:(3):208-213

[11]. Lee B, Ludlow B. Attitude of the Korean dentists towards radiation safety and selection criteria. Imaging Sci Dent. 2013;43:179-84

[12]. Kasat V, Ladda R, Joshi S, Giri PA, Pandya M, Shaikh S. Knowledge and practice regarding safety standards of oral radiology among dental practitioners in western Maharashtra, India. Oral Radiology 2014; 1:1-7.

[13]. Salti L, Whaites E. Survey of dental radiographic services in private dental clinics in Damascus, Syria. Dentomaxillofacial Radiology 2002;31:100-5

[14]. Aps J. Flemish general dental practitioners' knowledge of dental radiology. Dentomaxillofacial Radiol. 2010; 39:113-118

[15]. Shahab S, Kavosi A, Nazarinia H, Mehralizadeh S, et al. Compliance of Iranian dentists with safety standards of oral radiology. Dentomaxillofac Radiol. 2012;41:159-64.

[16]. Haghanifar S, Moudi E, Abesi F, Bijani A, Kazemi O. A Study of Dentists' Knowledge about Dental Radiology Principles. J Babol Univ Med Sci. 2015; 17:34-8.

[17]. Razi T, Ghojazadeh M, Bazvand L. Diagnostic dental radiation risk during pregnancy: awareness among general dentists in Tabriz. Journal of Dental Research, Dental Clinics, Dental Prospects 2011;5:67-70.

[18]. Jacobs R, Vanderstappen M, Bogaerts R, Gijbels F. Attitude of the Belgian dentist population towards radiation protection. Dentomaxillofacial Radiol. 2004;33:334-9.

[19]. chauDhry M, JayapraKaSh K, ShivalingeSh K, Agarwal V, Gupta B, Anand R, et al. Oral Radiology Safety Standards Adopted by the General Dentists Practicing in National Capital Region (NCR). Journal of Clinical and Diagnostic Research. 2016;10:42-45.

[20]. Orafi I, Rushton V. A questionnaire study to derive information on the working environment, clinical training, use of ancillary staff and optimization of patient radiation dose within UK dental practice. Int Endod J. 2012;45:763-72.

[21]. Prabhat M , Sudhakar S , Kumar B, Ramaraju. Knowledge, attitude and perception (KAP) of dental undergraduates and interns on radiographic protection- A questionnaire based cross-sectional study. Journal of Advanced Oral Research. 2011;2:45-50.

Table (1): Knowledge, awareness and practice of general dentists regarding oral radiology protective guidelines

\begin{tabular}{|l|l|l|l|}
\hline Knowledge, awareness and practice regarding X. ray protective guidelines & \multicolumn{1}{|c|}{ Yes } & No & I don't know \\
\hline Awareness of ALARA principle & $52(26.3 \%)$ & $117(59.1 \%)$ & $29(14.6 \%)$ \\
\hline $\begin{array}{l}\text { Awareness of National Council on Radiation Protection and International } \\
\text { Commission on Radiological Protection recommendations. }\end{array}$ & $59(29.8 \%)$ & $114(57.6 \%)$ & $25(12.6 \%)$ \\
\hline Awareness of usefulness of collimators and filters in dental radiography. & $102(51.5 \%)$ & $78(39.4 \%)$ & $18(9.1 \%)$ \\
\hline Knowledge of digital radiography requires less exposure than conventional. & $132(66.7 \%)$ & $34(17.2 \%)$ & $32(16.1 \%)$ \\
\hline Knowledge of high speed film requires a reduced exposure. & $82(41.4 \%)$ & $44(22.2 \%)$ & $72(36.4 \%)$ \\
\hline Practice of holding the films during exposure. & $34(17.1 \%)$ & $152(76.8 \%)$ & $12(6.1 \%)$ \\
\hline Use of lead aprons on a regular basis. & $41(20.7 \%)$ & $137(69.2 \%)$ & $20(10.1 \%)$ \\
\hline
\end{tabular}

* "as low as reasonably achievable"

Table (2): Association between knowledge and awareness of general dental practitioners and gender towards xray exposure

\begin{tabular}{|c|c|c|c|c|}
\hline & & \multicolumn{2}{|c|}{ Gender } & \multirow[b]{2}{*}{$P$. value } \\
\hline Knowledge \& awareness toward X ray exposure & Response & Male & Female & \\
\hline \multirow{3}{*}{ Dental $\mathrm{x}$ - ray is harmful } & Yes & $30(61.2 \%)$ & $79(53.0 \%)$ & \multirow{3}{*}{0.583} \\
\hline & No & $18(36.7 \%)$ & $65(43.6 \%)$ & \\
\hline & I don't know & $1(2.0 \%)$ & $5(3.4 \%)$ & \\
\hline \multirow{3}{*}{ X-ray beams reflect from room walls } & Yes & $24(49.0 \%)$ & $66(44.3 \%)$ & \multirow{3}{*}{0.434} \\
\hline & No & $17(34.7 \%)$ & $64(43.0 \%)$ & \\
\hline & I don't know & $8(16.3 \%)$ & $19(12.8 \%)$ & \\
\hline \multirow{3}{*}{ Aware of deterministic and stochastic effects } & Yes & $26(53.1 \%)$ & $65(43.6 \%)$ & \multirow{3}{*}{0.087} \\
\hline & No & $19(38.8 \%)$ & $61(40.9 \%)$ & \\
\hline & I don't know & $4(8.2 \%)$ & $23(15.4 \%)$ & \\
\hline \multirow{3}{*}{$\begin{array}{l}\text { Dental radiography absolutely contraindicated in } \\
\text { pregnant patients? }\end{array}$} & Yes & $9(18.4 \%)$ & $37(24.8 \%)$ & \multirow{3}{*}{0.650} \\
\hline & No & $38(77.6 \%)$ & $109(73.2 \%)$ & \\
\hline & I don't know & $2(4.1 \%)$ & $3(2.0 \%)$ & \\
\hline
\end{tabular}


Table 3: Association between knowledge of general dental practitioners about x-ray exposure and years of experience.

\begin{tabular}{|c|c|c|c|c|c|}
\hline & & \multicolumn{3}{|c|}{ Year of experience } & \multirow[b]{2}{*}{$P$ value } \\
\hline $\begin{array}{l}\text { Knowledge \& awareness about } \mathrm{X} \text { ray } \\
\text { exposure }\end{array}$ & & Less than a year & 1- 2 year & \begin{tabular}{|l} 
More than 2 \\
years
\end{tabular} & \\
\hline \multirow{3}{*}{ Is dental $\mathrm{x}$ - ray harmful? } & Yes & $25(41.7 \%)$ & $51(55.4 \%)$ & $33(71.7 \%)$ & \multirow{3}{*}{$0.029 *$} \\
\hline & No & $33(55.0 \%)$ & $37(40.2 \%)$ & $13(28.3 \%)$ & \\
\hline & I don't know & $2(3.3 \%)$ & $4(4.3 \%)$ & $0(0 \%)$ & \\
\hline \multirow{3}{*}{ X- Ray beams reflect from room walls? } & Yes & $22(36.7 \%)$ & $41(44.6 \%)$ & $27(58.7 \%)$ & \multirow{3}{*}{$0.039 *$} \\
\hline & No & $24(40.0 \%)$ & $42(45.7 \%)$ & $15(32.6 \%)$ & \\
\hline & I don't know & $14(23.3 \%)$ & $9(9.8 \%)$ & $4(8.7 \%)$ & \\
\hline \multirow{3}{*}{ X-ray cause ionization to matter? } & Yes & $22(36.7 \%)$ & $47(51.1 \%)$ & $27(58.7 \%)$ & \multirow{3}{*}{$0.049 *$} \\
\hline & No & $16(26.7 \%)$ & $18(19.6 \%)$ & $13(28.3 \%)$ & \\
\hline & I don't know & $22(36.7 \%)$ & $27(29.3 \%)$ & $6(13.0 \%)$ & \\
\hline \multirow{3}{*}{$\begin{array}{l}\text { Aware of deterministic and stochastic effects } \\
\text { of } X \text { ray radiation }\end{array}$} & Yes & $23(38.3 \%)$ & $42(45.7 \%)$ & $26(56.5 \%)$ & \multirow{3}{*}{$0.050 *$} \\
\hline & No & $24(40.0 \%)$ & $37(40.2 \%)$ & $19(41.3 \%)$ & \\
\hline & I don't know & $13(21.7 \%)$ & $13(14.1 \%)$ & $1(2.2 \%)$ & \\
\hline \multirow{3}{*}{$\begin{array}{l}\text { Is dental radiograph contraindicated in } \\
\text { pregnant patients? }\end{array}$} & Yes & $6(10.0 \%)$ & $26(28.3 \%)$ & $14(30.4 \%)$ & \multirow{3}{*}{$0.032 *$} \\
\hline & No & $51(85.0 \%)$ & $64(69.6 \%)$ & $32(69.6 \%)$ & \\
\hline & I don't know & $3(5.0 \%)$ & $2(2.2 \%)$ & $0(.0 \%)$ & \\
\hline \multirow{3}{*}{ Aware of ALARA* principle } & Yes & $9(15.0 \%)$ & $23(25.0 \%)$ & $20(43.5 \%)$ & \multirow{3}{*}{$0.022 *$} \\
\hline & No & $41(68.3 \%)$ & $54(58.7 \%)$ & $22(47.8 \%)$ & \\
\hline & I don't know & $10(16.7 \%)$ & $15(16.3 \%)$ & $4(8.7 \%)$ & \\
\hline
\end{tabular}

*“as low as reasonably achievable” 